\title{
Research Paper \\ Association between Interleukin 16 Gene Polymorphisms (rs1131445, rs4072111) and Late Onset of Alzheimer's Disease in Iranian Patients
}

\author{
Nazanin Esmaeili Anvar ${ }^{1}$, Niloofar Bazazzadegan ${ }^{1}$, Mina Ohadi ${ }^{1}$, Kourosh Kamali ${ }^{2}$, ${ }^{*}$ Hamid Reza Khorram Khorshid ${ }^{1}$
}

1. Genetic Research Center, University of Social Welfare and Rehabilitation Sciences, Tehran, Iran.

2. Department of Genetics and Biotechnology Reproduction, Reproductive Biotechnology Research Center, Avicenna Research Institute, Tehran, Iran.

Crtation: Esmaeili Anvar N, Bazazzadegan N, Ohadi M, Kamali K, Khorram Khorshid HR. [Study of association between interleukin 16 gene polymorphisms (rs1131445, rs4072111) and late onset Alzheimer disease in Iran (Persian)]. Iranian Journal of Ageing. 2016; 11(4):64-71. http://dx.doi.org/10.21859/sija-110164

doi): http://dx.doi.org/10.21859/sija-110164

Received: 04 Oct. 2015 Accepted: 31 Jan. 2016

Key words: Cardiovascular, Alzheimer, Polymorphisms, Cytokines, Interleukin 16

\section{A B STRACT}

Objectives Alzheimer's disease is the most common kind of dementia in the old age. Previous studies have shown that cytokines are proteins that play an important role in inflammation. It may be responsible for inflammatory pathology seen around old age plaques in Alzheimer's disease. It can also associate with the pathology of Alzheimer's disease. Therefore, the aim of this study was to investigate the effects of 2 polymorphisms of gene IL16 (rs1131445 and rs4072111) on the risk of patients diagnosed with Alzheimer's disease in the Iranian population.

Methods \& Materials In this study, the intervention group consisted of 144 individuals who were diagnosed with Alzheimer's disease by psychologists based on a clinical test (DSM-IV), and the control group included 173 healthy individuals with no psychological disorders. DNA was extracted by salting out technique. The PCR response was conducted (for replicating the mentioned pieces) for any polymorphism in optimized conditions by using designed primers. The product of PCR was first checked for the confirmation of accurate function of PCR using polyacrylamide gel electrophoresis (PAGE). Next, the PCR product was dissected by restriction fragment length polymorphism (RFLP) method with intended confining enzyme, and then the genotype of the samples was determined by PAGE. Individual genotypes were determined using the PCR-RFLP method. Statistical analyses were done using OpenEpi 2.3.1 and SPSS 11.5. Results The study of allelic analysis between the control and intervention groups by considering the confidence interval ( $\mathrm{Cl}=90 \%)$ and significant level (0.05) for rs1131445 showed that the $\mathrm{C}$ allele had no significant association with Alzheimer's disease $(P=0.656)$. The TC genotype did not show any significant difference with $\Pi$ genotype $(P=0.614)$. However, the study of allelic analysis for $r 54072111$ polymorphism between 2 groups showed that the relationship of $\mathrm{T}$ allele with the disease is significant, and this allele has a protective role in creating the disease $(\mathrm{P}=0.008)$. In addition, TC genotype as a protective status showed a significant association with Alzheimer's disease ( $P=0.007)$.

Conclusion The existence of polymorphism in some genes of the inflammatory pathway could make people susceptible to Alzheimer's disease. The genetic changes in DNA sequence of gene IL16 could result in changes in cytokine product or its function. The association between rs4072111 and Alzheimer's disease supports the presumptions and shows a probable role of this polymorphism in Alzheimer disease. Besides, the association between rs1131445 and Alzheimer disease cannot be proven due to the small number of samples (Power: 8.23\%).

\section{* Corresponding Author: \\ Hamid Reza Khorram Khorshid, MD, PhD}

Address: Genetic Research Center, University of Social Welfare and Rehabilitation Sciences, Kodakyar Ave., Daneshjo Blv., Evin, Tehran, Iran. Tel: +98 (21) 22180138

E-mail: hrkk1@uswr.ac.ir 


\title{
بررسى ارتباط هيلىمورفيسمهاى rs1131445 و rs4072111 ثن مسير التهابى اينترلوكين وا با با بيمارى آلزايمر دير آبل دور در ايران
}

\author{
نازنين اسمعيلىانور'، نيلوفر بزاززادكان'، مينا اوحدى'؛ كوروش كمالى ؛ "حميدرضا خرمخورشيد'

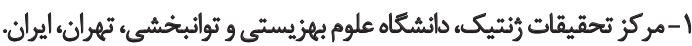

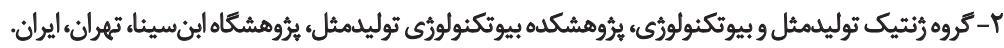

\begin{abstract}
حكSد

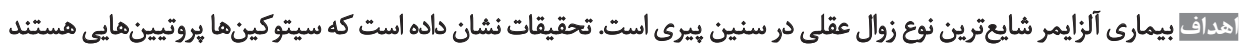

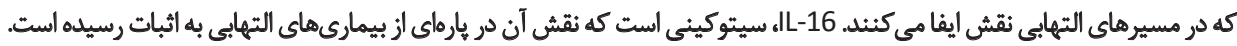

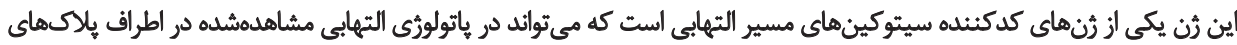

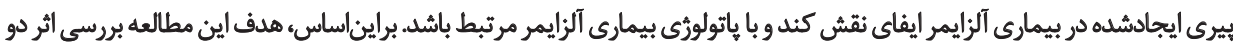

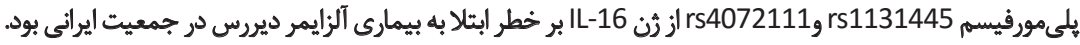

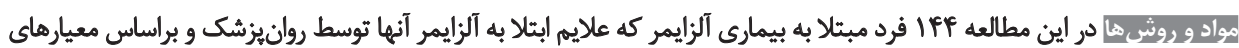

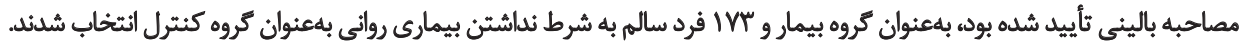

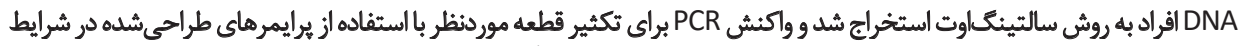

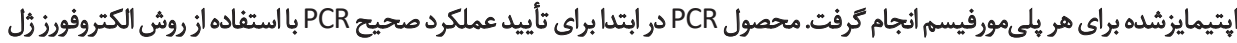

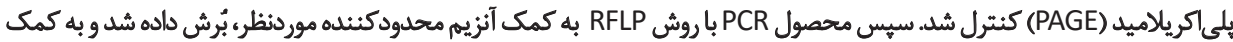

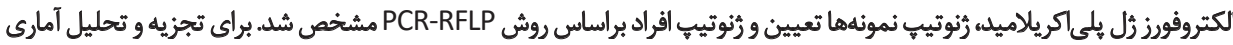

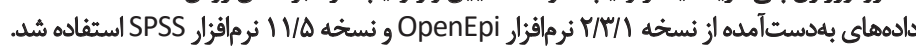

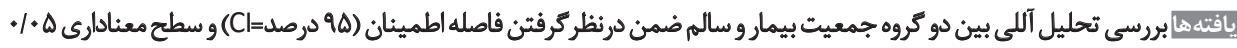

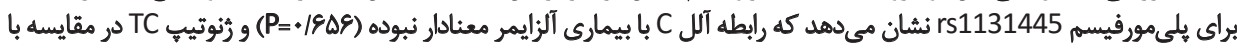

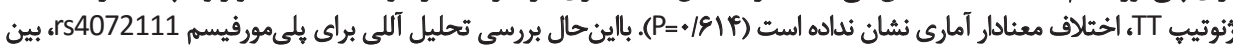

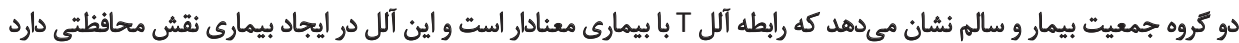

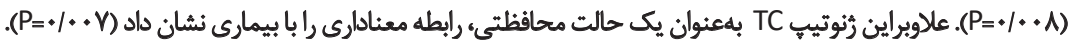

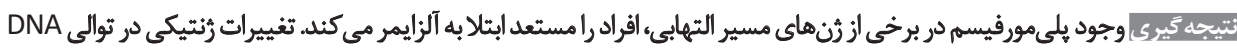

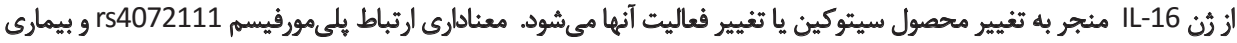

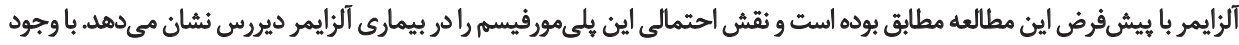

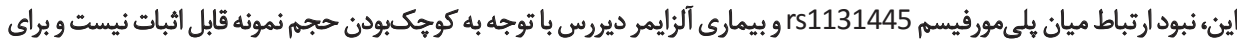

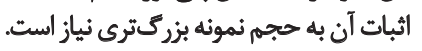

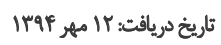

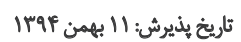

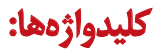

قلب و عروق، آلزايمر،

يلي مورفيسمهاء سيتوكينها، اينتر لوكين

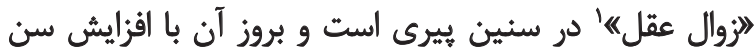

مقدمه

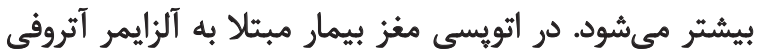

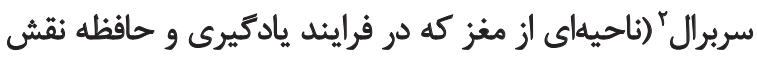

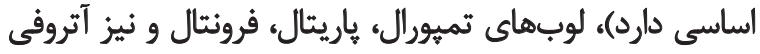

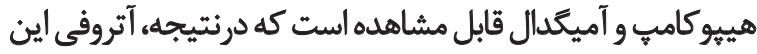

بيمارى آلزايمر شايعترين عامل زوال عقل در دوران ميانسالى في في في

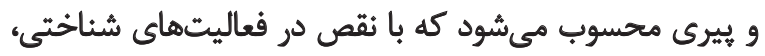

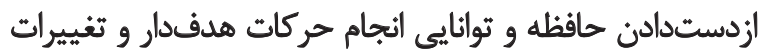
شخصيتى همراه است. بيمارى آلزايمر كستردهترين نوع دمانس

1. Dementia

2. Cerebral atrophy

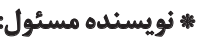
دكتر حميدرضا خرم مخور شيديد

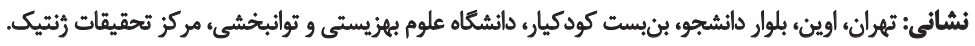
تلفن: يست الكثرونيكي: hrkk1@uswr.ac.ir 


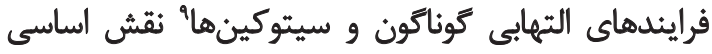

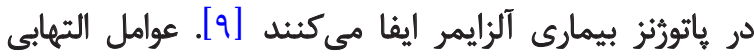

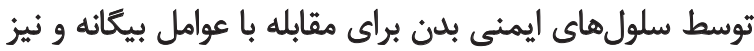

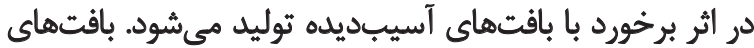

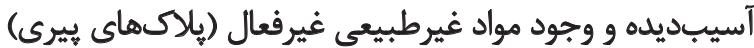

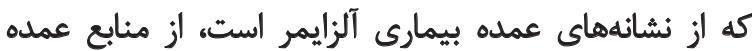

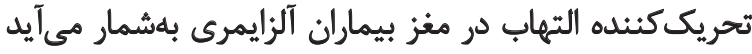

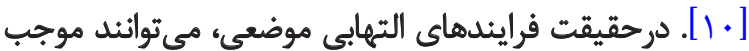

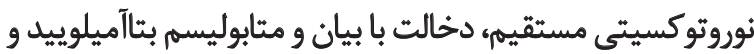

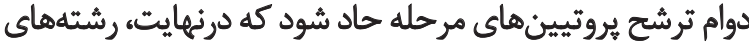

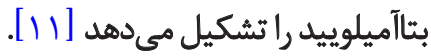

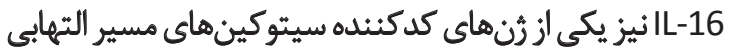

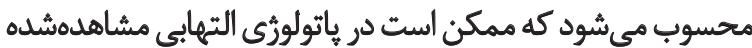

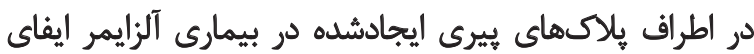

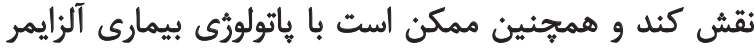

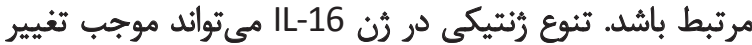

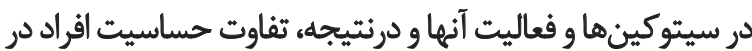

$$
\text { بيمارىهاي مختلف شود. }
$$

رثن كدكنيده L-16 روى كروموزوم 15q25/1 قرار كرفته است

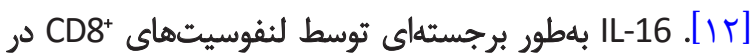

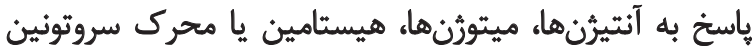

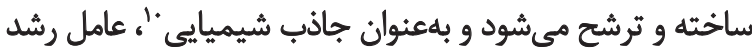

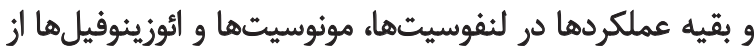

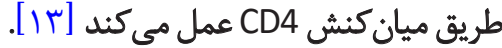
نقش IL-16 در بيمارىهاى مختلف التهابي مشخص شده

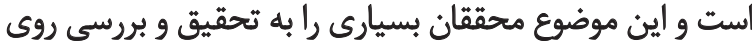

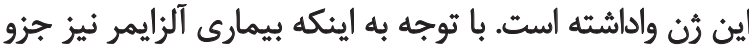

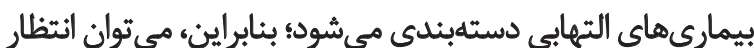

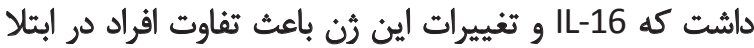

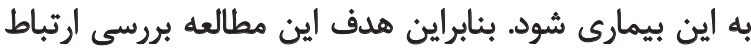

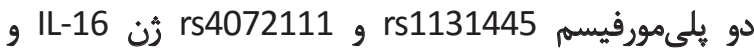
بيمارى آلزايمر ديررس در جمعيسم ايراني است. بلى إمورفيسم

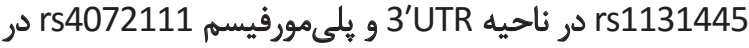

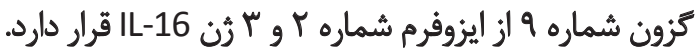

$$
\text { ورث مطالعه }
$$

در اين يروهش، نمونههاى خون افراد بيمار و سالم از

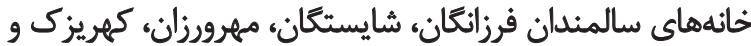

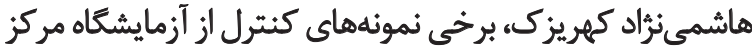

9. Cytokines

10. Chemo attractant
نواحى نقص بيشرونده حافظه ب، اختلال شناختى" و و تغييرات شخصيتى ه رادر فرد مبتلا بهوجود هى آورد [ [ و ا I]

علايم اين بيمارى با ازدستدادن قدرت حفظ اطلاعات بهويره

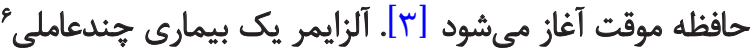

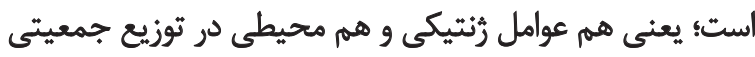

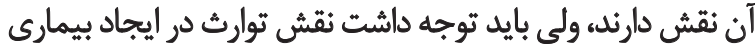

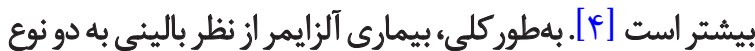

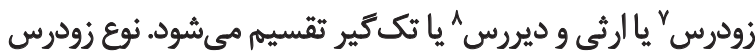

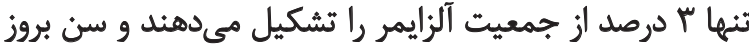

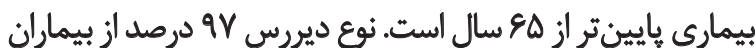

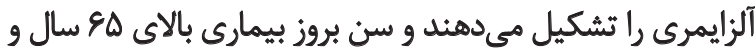
تكگير است [ه] و اين مطالعه روى اين نوع از بيمارى تمركز دارد. با توجه به بهبود وضعيت زندكى و افزايش طول عمر، در

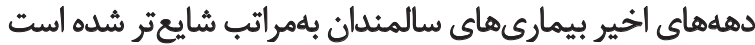

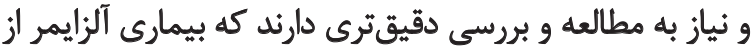

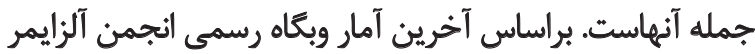

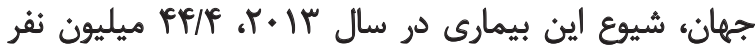

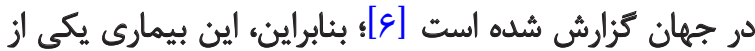

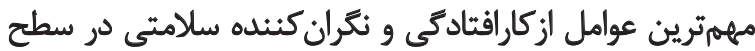

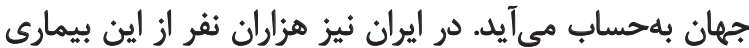

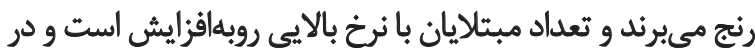

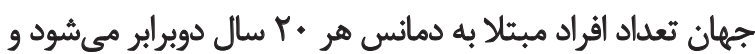

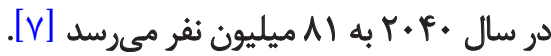

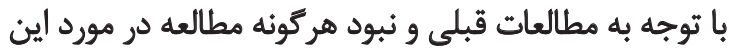

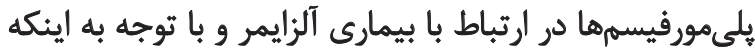

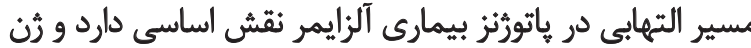

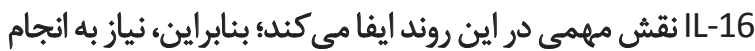

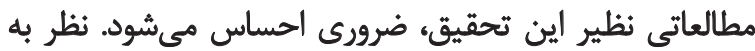

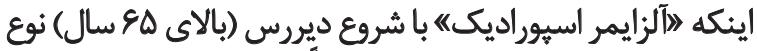

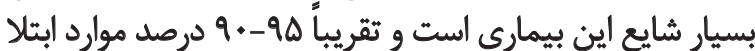

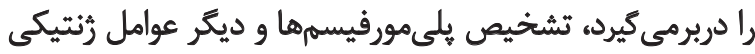

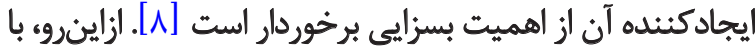

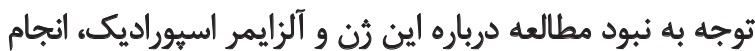

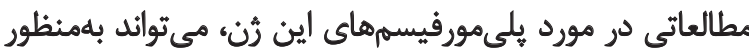
بيشكيرى و تشخيص بيمارى مورد استفاده قرار تيرد.

3. Progressive memory deficit

4. Cognitive impairment

5. Personality changes

6. Multi factorial

7. Early-onset

8. Late-onset 


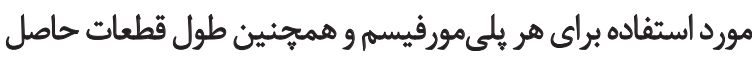

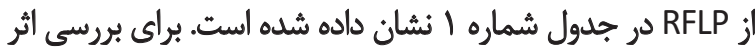

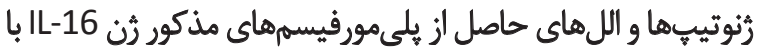

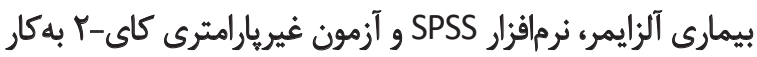
كرفته شد.

L. 1.

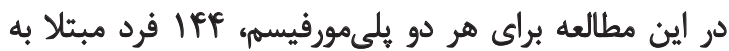

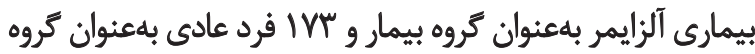

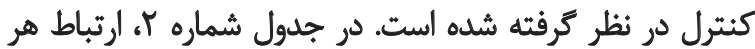

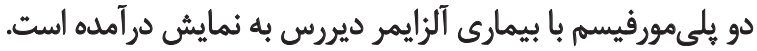

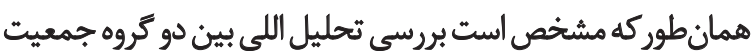

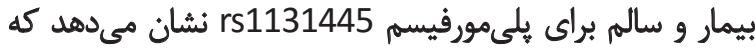

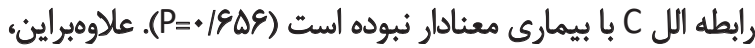

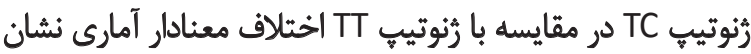

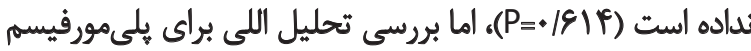

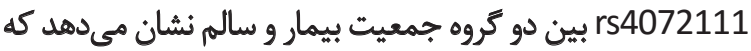

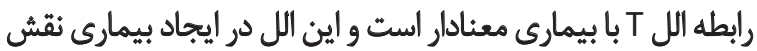

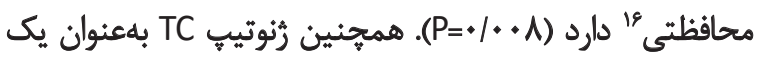

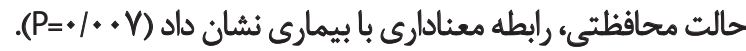
اثر متقابل زنوتيڤها و اللها از نظر جنسيت نيز براى هر دو

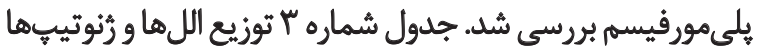

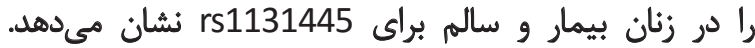

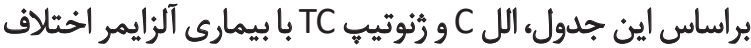

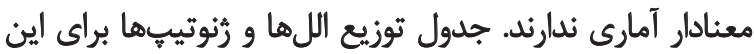

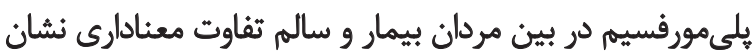

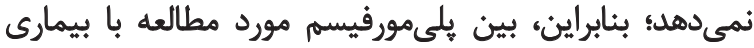

16. Protective
رماتيسم ايران و بسيارى از نمونههاى بيماران از انجمن آلرايمر

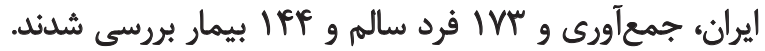

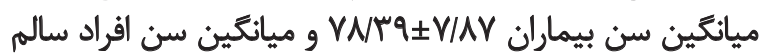

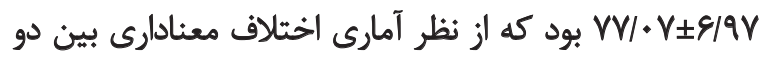

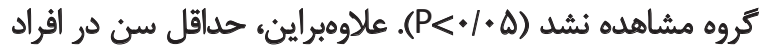

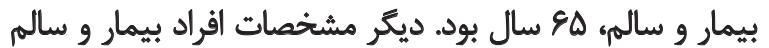

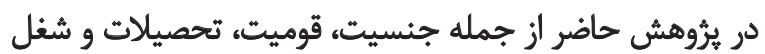

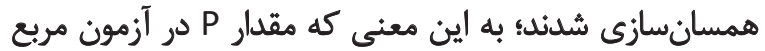
خي-ז، در هيجيك از سطوح معنادار نبود.

تشخيص بيمارى در بيماران توسط كروهى از روانيزشكان با بان

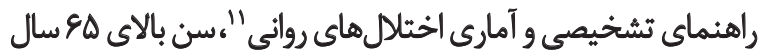

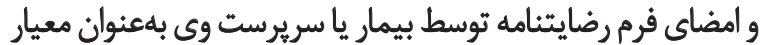

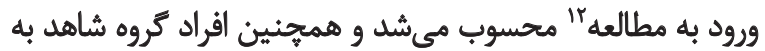

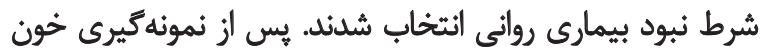

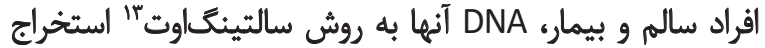

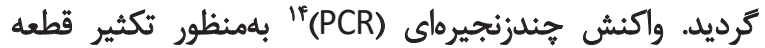

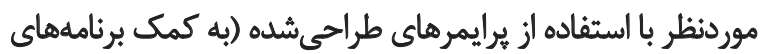

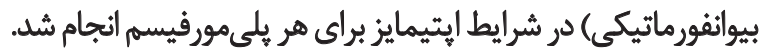

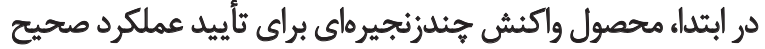

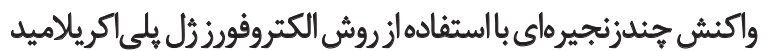

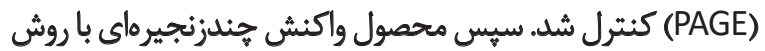

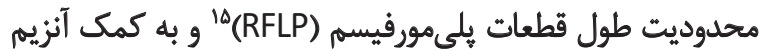

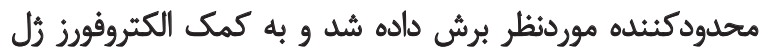

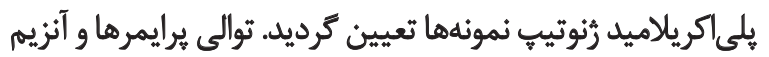

11. Diagnostic and Statistical Manual of Mental Disorders (DSM-IV) 12. Inclusion Criteria

13. Salting out

14. Poly Chain Reaction

15. Restriction Fragment Length Polymorphism

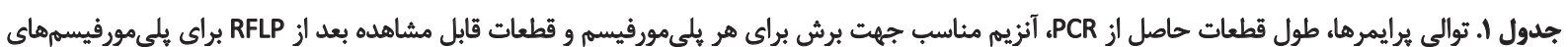
.rs4072111, rs1131445

\begin{tabular}{|c|c|c|c|c|c|}
\hline أندازه قطعات RFLP & (آنزيم محدودكنينده انكوباسيون) & $\begin{array}{c}\text { اندازه قطعات حاصل } \\
\text { أن (bp) }\end{array}$ & توالى يرايمر & نوكلئوتيد & بلى مورفسيم \\
\hline $\begin{array}{c}T=r M \mid \\
C=|Q \cdot+Y|\end{array}$ & $\operatorname{BsaAl}\left(\mu \cdot{ }^{\circ} \mathrm{C}\right)$ & MT & 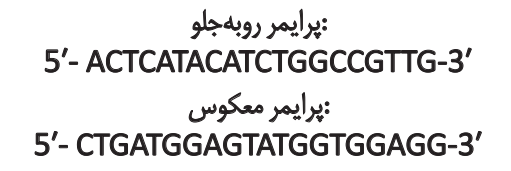 & $(\mathrm{T} / \mathrm{C})$ & rs1131445 \\
\hline $\begin{array}{c}C=19 f \\
T=\mid f \cdot+r f\end{array}$ & $\operatorname{BsmAl}\left(r^{\circ} \mathrm{C}\right)$ & ief & $\begin{array}{c}\text { 5' يرايمر روبهجلو: } \\
\text { 5'- CACTGTGATCCCGGTCCAGTC-3' } \\
\text { 5'- TTCAGGTACAAACCCAGCCAGC -3' }\end{array}$ & $(C / T)$ & rs4072111 \\
\hline
\end{tabular}

L 


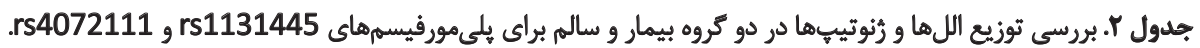

\begin{tabular}{|c|c|c|c|c|c|}
\hline OR (Cl=9๑\%) & P-value & $\begin{array}{c}\text { تعداد افراد كتّرل } \\
\text { N (\%) }\end{array}$ & $\begin{array}{c}\text { تعداد الفراد بيمار } \\
\text { N (\%) }\end{array}$ & مثغير & بلى مورفيسم \\
\hline & & & & رثوتيبي & \multirow{7}{*}{ rs1131445 } \\
\hline \multicolumn{2}{|c|}{ كروه مرجع } & $Q Y(\Delta r / \varepsilon)$ & $A r(\Delta S / 9)$ & $\pi$ & \\
\hline$\cdot \mid A N(\cdot / \Delta P-1 / N)$ &.$|8| f$ & $e q(p \cdot 1)$ & $\Delta f(M V(\Delta)$ & $\mathrm{TC}$ & \\
\hline$\cdot \mid A Y(\cdot / M Y-Y / M)$ & . Isqu & $M(\varepsilon / \pi)$ & $\Lambda(\Delta / \&)$ & $\mathrm{CC}$ & \\
\hline & & & & اللل - الل & \\
\hline \multicolumn{2}{|c|}{ كروه مرجع } & $r \Delta \Delta\left(M^{\prime} / 8\right)$ & MIANO/F) & $\mathbf{T}$ & \\
\hline$\cdot / 9(\cdot / 8-1 / 4)$ & $.1 \Delta \& \Delta$ & $91(Y \& / P)$ & $V \cdot(M+p)$ & C & \\
\hline \multirow{2}{*}{\multicolumn{2}{|c|}{ كروه مرجع }} & & & رئوتيبي & \multirow{7}{*}{ rs4072111 } \\
\hline & & $\pi r \cdot(e q / \Delta)$ & $119(A T / V)$ & $\mathrm{CC}$ & \\
\hline$+/ \uparrow \lambda(+/ Y \Lambda-+/ A \mu)$ & $\%+V$ & $\Delta r(r+)$ & $r \Delta(I V / T)$ & $\mathrm{TC}$ & \\
\hline Unidentified & $\begin{array}{c}1 \text { Fisher exact test } \\
\text { P-value }\end{array}$ & $1(\cdot / 0)$ & $\cdot(\cdot)$ & $T T$ & \\
\hline & & & & اللال - ال & \\
\hline \multicolumn{2}{|c|}{ كروه مرجع } & $\operatorname{rar}(A F / F)$ & rerr(q)/f) & C & \\
\hline$\cdot(\Delta)(\cdot / \mu)-+\mid \Delta \Delta)$ & $+1+\cdots$ & $\Delta f(I \Delta / S)$ & $r \Delta(N E)$ & $\mathrm{T}$ & \\
\hline
\end{tabular}

L

IL-16 و بيمارى آلزايمر انجام نشده است، اما با توجه به اينكه

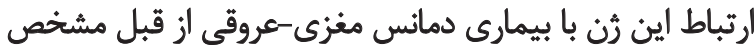

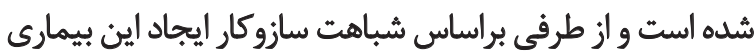

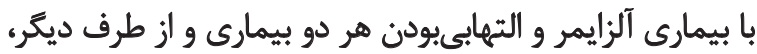

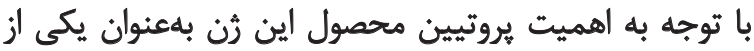

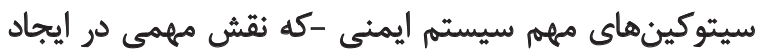

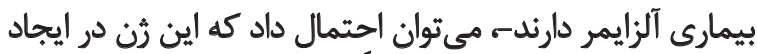

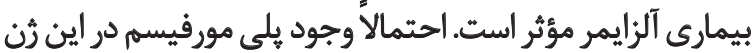

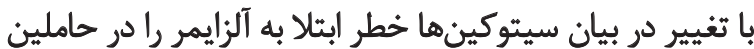

$$
\text { اين يلى مورفيسمها متفاوت ميى كند. }
$$

تاكنون جند مطالعه به بررسى ارتباط يلى مورفيسمهاى اين

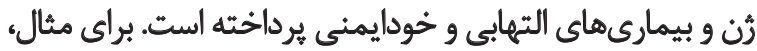

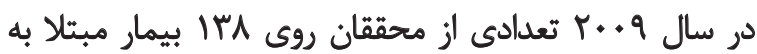

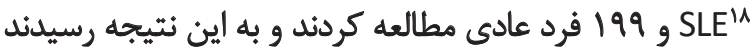

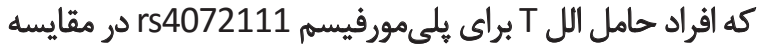

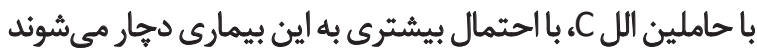

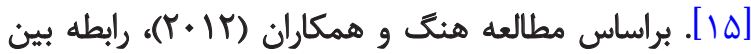

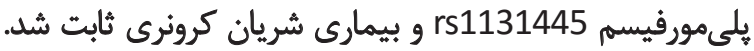

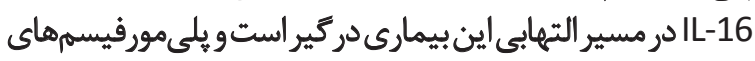

آلزايمر ديررس در مردان بيمار و سالم، تفاوت معنادارى ديده نشد.

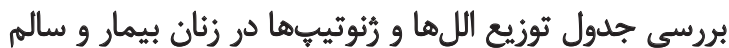

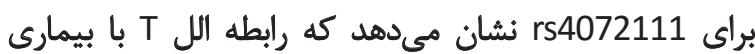

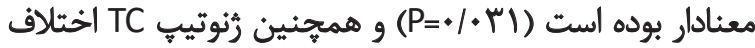

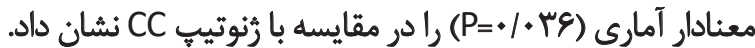

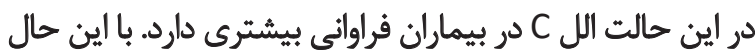

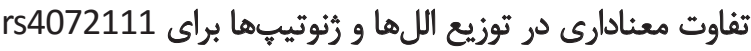
در بين مردان بيمار و سالم ملاحظه نترديد. $\stackrel{\leftrightarrow}{\oplus}$

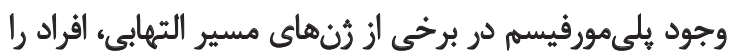

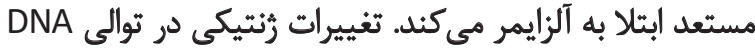

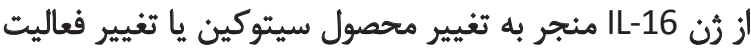

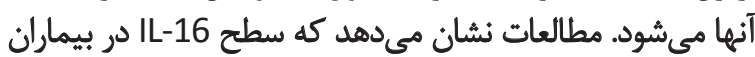

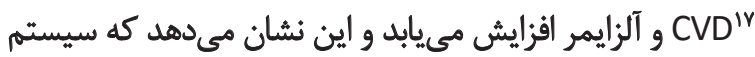
ايمنى نقش مهمى را در ناهنجارى مهاي نورودرئريتيو ايفا مي كئد

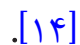

تاكنون هيج مطالعهاى درباره بررسى يلىمورفيسمهاى ثن

17. Cerebral Vascular Dementia 


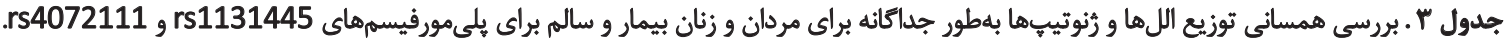

\begin{tabular}{|c|c|c|c|c|c|c|c|}
\hline \multicolumn{3}{|c|}{ ز } & \multicolumn{3}{|c|}{ مرد } & \multirow[t]{2}{*}{ هتغير } & \multirow[t]{2}{*}{ يلى مورفيسم } \\
\hline OR (৭৯\%Cl), P-value & كيترل & بيمار & OR ( $৭ ৯ \% \mathrm{Cl}), \mathrm{P}$-value & كتثرل & ل بيمار & & \\
\hline & & & & & & 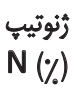 & \\
\hline كروه مرجع & $\Delta r(\Delta r)$ & $r N(\Delta r / \Delta)$ & كروه مرجع & $r q(\Delta \Delta / F)$ & $P(F I / Y)$ & $\pi$ & \\
\hline $1(\cdot / \Delta-1 / 9)_{6}+/ 99$ & $r q(p q)$ & $r A(r q / r)$ & $\cdot / N A(\cdot / r A-V / \Delta A) \cdot+/ F q r$ & $r V(F) / \Delta)$ & $M F(T \Delta / \Lambda)$ & TC & \\
\hline$\cdot / A V(+/ T E-r / \Lambda) \cdot / A V$ & $N(A)$ & $\Delta(Y / \nu)$ & $+/ 11+/ 11-8 / \Delta) \sigma \cdot / 199$ & $r(\Psi / N)$ & $r(\mu)$ & $\mathrm{CC}$ & rs1131445 \\
\hline & & & & & & $\begin{array}{c}\text { الل } \\
\mathbf{N}(\%)\end{array}$ & \\
\hline كروه هرجع & $1 f Q(N / \Delta)$ & $1 . f(M / T)$ & كروه مرجع & $99(18 / 1)$ & $1.8(M / 1)$ & $\mathrm{T}$ & \\
\hline$. / 98(\cdot / 8-1 / 8)=\cdot / 179$ & $\Delta Q(T V / \Delta)$ & TARE/A) & $\cdot / \Delta F \Delta(\cdot / P V-1 / \Delta), \cdot / A F$ & $r)(\pi / 9)$ & $r A(T \cdot / 9)$ & C & \\
\hline & & & & & & 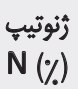 & \\
\hline كروه مرجع & $V \cdot(V+)$ & $8 \cdot(A F / \Delta)$ & كروه مرجع & $P V(N / T)$ & $\Delta r(V / /)$ & $\mathrm{CC}$ & \\
\hline 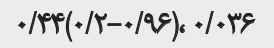 & rq(rq) & $M(1 \Delta / \Delta)$ & $1 / F \Delta(+/ \& \Delta-T / T)_{k}+/ T E T$ & $M(T V / V)$ & $I F(T+/ 9)$ & $\mathrm{TC}$ & \\
\hline Unidentified & $1(1)$ & $*(*)$ & Unidentified & $+(*)$ & $+(*)$ & $\pi$ & rs4072111 \\
\hline .148 & & & $1 / 8$ & & & 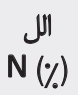 & \\
\hline كروه مرجع & $1 E q(A F / \Delta)$ & $|r|(94 / T)$ & كروه مرجع & $\operatorname{QP}(N \in \mathbb{T})$ & $M+(A q / Y)$ & c & \\
\hline$\cdot / P \&(\cdot / M Y-\cdot / Q Y)_{*} \cdot 1 \cdot H_{1}$ & $M(\mid \Delta / \Delta)$ & $M(N / N)$ & $1 / F(\cdot / N A-r / \Delta)_{k} \cdot / 19 r$ & $M(I r / A)$ & $1 f(1 \cdot / 4)$ & $\mathbf{T}$ & \\
\hline
\end{tabular}

Lـ

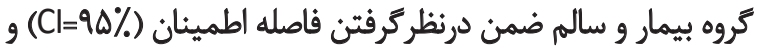

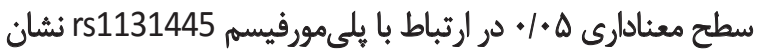

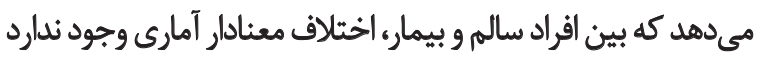
كه با توجه به حجم كم نمونهها (Power=ATY\%) براى اثبات اين

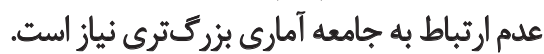

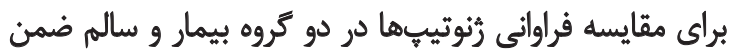
درنظركرفتن فاصله اطمينان (

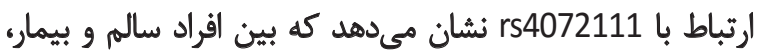

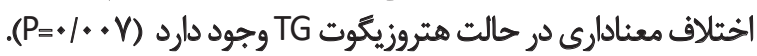

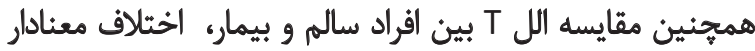

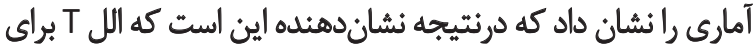

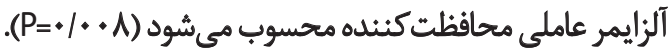

\section{نتيجليَيرى نهايى}

با توجه به نقش غيرقابل انكار بلى مورفيسمها در بيمارىهاى إئي

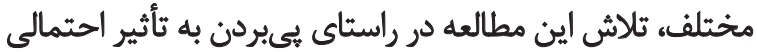

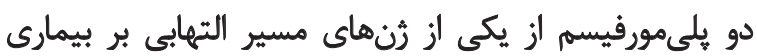

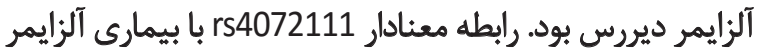

مختلف آن، احتمال ابتلا به اين بيمارى راتغيير ميدهند [91].

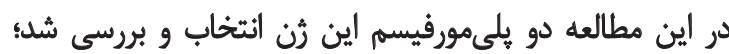

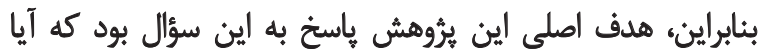

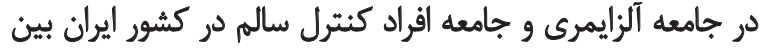

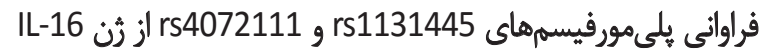

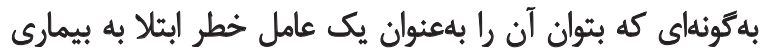

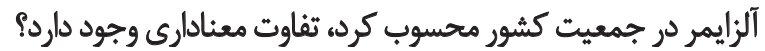
درآينده، با فزايش اطلاعات حاصل از اين كونه مطالعات وبادر اختيار

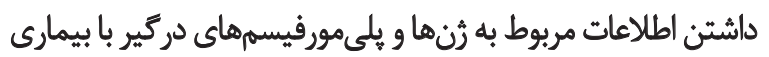

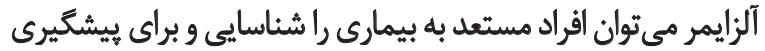

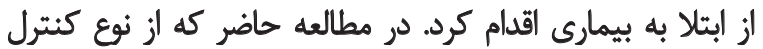

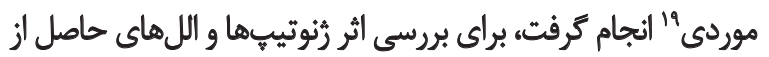

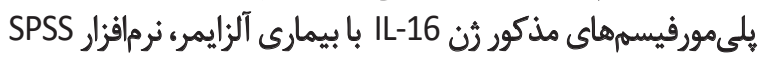

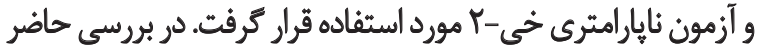

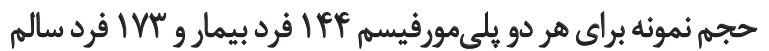

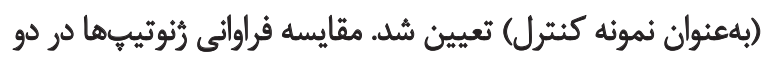

19. Case-control 


\section{References}

[1] Kaida KI, Takeda K, Nagata N, Kamakura K. Alzheimer's disease with asymmetric parietal lobe atrophy: A case report. Journal of the Neurological Sciences. 1998; 160(1):96-9.

[2] Heneka MT, O'Banion MK. Inflammatory processes in Alzheimer's disease. Journal of Neuroimmunology. 2007; 184(1):69-91.

[3] Szymański P, Markowicz M, Janik A, Ciesielski M, MikiciukOlasik E. Neuroimaging diagnosis in neurodegenerative diseases. Nuclear Medicine Review. 2010; 13(1):23-31.

[4] Araria-Goumidi L, Lambert JC, Cottel D, Amouyel P, ChartierHarlin MC. No association of the HLA-A2 allele with Alzheimer's disease. Neuroscience Letters. 2002; 335(2):75-8.

[5] St George-Hyslop PH, Petit A. Molecular biology and genetics of Alzheimer's disease. Comptes Rendus Biologies. 2005; 328(2):11930.

[6] Dementia statistics [Internet]. 2014 [Cited 08 Nov. 2014]. Available from: http://www.alz.co.uk/research/statistics.

[7] Ferri CP, Prince M, Brayne C, Brodaty H, Fratiglioni L, Ganguli $\mathrm{M}$, et al. Global prevalence of dementia: A Delphi consensus study. Lancet. 2006; 366(9503):2112-7.

[8] Bertram L, Tanzi RE. Alzheimer's disease: One disorder, too many genes? Human Molecular Genetics. 2004; 13(1):135-41.

[9] Greig NH, Mattson MP, Perry T, Chan SL, Giordano T, Sambamurti K, et al. New Therapeutic Strategies and Drug Candidates for Neurodegenerative Diseases: p53 and TNF-a Inhibitors, and GLP-1 Receptor Agonists. Annals of the New York Academy of Sciences. 2004; 1035(1):290-315.

[10] Akiyama H, Barger S, Barnum S, Bradt B, Bauer J, Cole GM, et al. Inflammation and Alzheimer's disease. Neurobiology of Ageing. 2000; 21(3):383-421.

[11] Terreni L, Fogliarino S, Quadri P, Ruggieri RM, Piccoli F, Tettamanti M, et al. Tumor necrosis factor a polymorphism C-850T is not associated with Alzheimer's disease and vascular dementia in an Italian population. Neuroscience Letters. 2003; 344(2):135-7.

[12] Gao LB, Liang WB, Xue H, Rao L, Pan XM, Lv ML, et al. Genetic polymorphism of interleukin-16 and risk of nasopharyngeal carcinoma. Clinica Chimica Acta. 2009; 409(1):132-5.

[13] Zhang Y, Center DM, David MH, Cruikshank WW, Yuan J, Andrews DW, Kornfeld H. Processing and activation of pro-interleukin-16 by caspase-3. Journal of Biological Chemistry. 1998; 273(2):1144-9.

[14] Di Rosa M, Dell'Ombra N, Zambito AM, Malaguarnera M, Nicoletti F, Malaguarnera L. Chitotriosidase and inflammatory mediator levels in Alzheimer's disease and cerebrovascular dementia. European Journal of Neuroscience. 2006; 23(10):2648-56.

[15] Xue H, Gao L, Wu Y, Fang W, Wang L, Li C, et al. The IL-16 gene polymorphisms and the risk of the systemic lupus erythematosus. Clinica Chimica Acta. 2009; 403(1):223-5

[16] Huang H, Zeng Z, Zhang L, Liu R, Li X, Qiang O, et al. The association of interleukin-16 gene polymorphisms with susceptibility of coronary artery disease. Clinical biochemistry. 2013; 46(3):241-4.

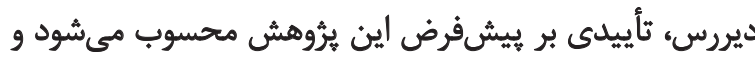

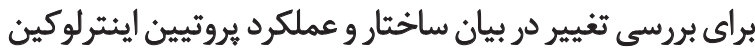

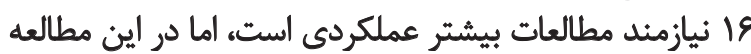

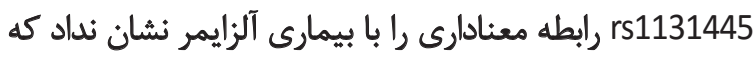

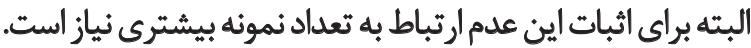

$$
\text { تشكر وقدردانى }
$$

نويسندكان اين مقاله مراتب قدرداني و تشكر خود رالز همكارى

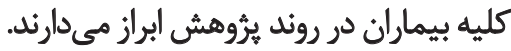


